OPEN ACCESS

Edited by:

Xing Tian,

New York University Shanghai, China

Reviewed by:

Dezhong Yao,

University of Electronic Science and

Technology of China, China

Jianhui Wu,

Institute of Psychology (CAS), China

*Correspondence:

Guoming Luan

luangm3@163.com

Qian Wang

aleinwangba@126.com

Shenglin She

15013144670@126.com

tThese authors have contributed equally to this work

Received: 09 August 2016 Accepted: 17 November 2016 Published: 28 November 2016

Citation:

Zheng Y, Li H, Ning Y, Ren J, Wu Z, Huang $R$, Luan G, Li T, Bi T, Wang $Q$ and She $S$ (2016) Sluggishness of

Early-Stage Face Processing (N170)

Is Correlated with Negative and

General Psychiatric Symptoms in

Schizophrenia.

Front. Hum. Neurosci. 10:615. doi: 10.3389/fnhum.2016.00615

\section{Sluggishness of Early-Stage Face Processing (N170) Is Correlated with Negative and General Psychiatric Symptoms in Schizophrenia}

\author{
Yingjun Zheng ${ }^{1 \dagger}$, Haijing $\mathrm{Li}^{1 \dagger}$, Yuping Ning ${ }^{1}$, Jianjuan Ren ${ }^{1}$, Zhangying $W u^{1}$, \\ Rongcheng Huang ${ }^{1}$, Guoming Luan ${ }^{2,3 *}$, Tianfu $\mathrm{Li}^{2,3}$, Taiyong Bi ${ }^{4,5}$, Qian Wang ${ }^{2 *}$ \\ and Shenglin She ${ }^{1 *}$ \\ ${ }^{1}$ Department of General Psychiatry, The Affiliated Brain Hospital of Guangzhou Medical University (Guangzhou Huiai \\ Hospital), Guangzhou, China, ${ }^{2}$ Beijing Key Laboratory of Epilepsy, Epilepsy Center, Department of Functional Neurosurgery, \\ Sanbo Brain Hospital, Capital Medical University, Beijing, China, ${ }^{3}$ Beijing Institute for Brain Disorders, Beijing, China, ${ }^{4}$ Key \\ Laboratory of Cognition and Personality (SWU), Ministry of Education, Chongaing, China, ${ }^{5}$ Faculty of Psychology, Southwest \\ University, Chongqing, China
}

Patients with schizophrenia consistently exhibit abnormalities in the N170 event-related potential (ERP) component evoked by images of faces. However, the relationship between these face-specific N170 abnormalities in patients with schizophrenia and the clinical characteristics of this disorder has not been elucidated. Here, ERP recordings were conducted for patients with schizophrenia and healthy controls. The amplitude and latency of the N170 component were recorded while participants passively viewed face and non-face (table) images to explore the correlation between face-specific processing and clinical characteristics in schizophrenia. The results provided evidence for a face-specific N170 latency delay in patients with schizophrenia. The N170 latency in patients with schizophrenia was significantly longer than that in healthy controls when images of faces were presented in both upright and inverted orientations. Importantly, the face-related N170 latencies of the left temporo-occipital electrodes (P7 and PO7) were positively correlated with both negative and general psychiatric symptoms in these patients. The N170 amplitudes were weaker in patients than in controls for inverted images of both faces and non-faces (tables), with a left-hemisphere dominance. The face inversion effect (FIE), meaning the difference in N170 amplitude between upright and inverted faces, was absent in patients with schizophrenia, suggesting an abnormality of holistic face processing. Together, these results revealed a marked symptom-relevant neural delay associated with face-specific processing in patients with schizophrenia, providing additional evidence to support the demyelination hypothesis of schizophrenia.

\section{Keywords: schizophrenia, N170, face processing, schizophrenia symptoms, demyelination}

\section{INTRODUCTION}

Schizophrenia is a psychiatric disorder that is marked by positive symptoms, negative symptoms, cognitive deficits, motor abnormalities and social dysfunction (for example see Onitsuka et al., 2013). One consistent cognitive dysfunction in people diagnosed with schizophrenia involves non-emotional face processing (for reviews see Darke et al., 2013; Onitsuka et al., 2013; 
Watson, 2013; Bortolon et al., 2015; McCleery et al., 2015) both at behavioral (Chen, 2011; Maher et al., 2015, 2016) and physiological levels (Herrmann et al., 2004; Onitsuka et al., 2006; Tsunoda et al., 2012; Maher et al., 2016).

Event-related potentials (ERPs) are cortical electrophysiological responses to the presentation of a stimulus. A negativity that peaks at around $170 \mathrm{~ms}$ (N170) after stimulus onset, which is markedly enhanced by faces rather than by objects (Bentin et al., 1996), has been localized mainly to the fusiform face area (Rossion et al., 2003). Face-specific N170 potentials are significantly reduced in people with schizophrenia (Herrmann et al., 2004; Onitsuka et al., 2006; Tsunoda et al., 2012; Maher et al., 2016), suggesting that they have basic perceptual deficits in face processing.

Theoretically, individuals process face holistically, which has been shown by the face inversion effect (FIE) in which face discrimination performance for inverted faces is reduced compared with that for upright faces (Yin, 1969, 1970; Bauser et al., 2012). Previous studies have shown that the behavioral FIE is reduced or even absent in people with schizophrenia (Shin et al., 2008; Kim et al., 2010; Bauser et al., 2012; Megreya, 2016), suggesting a configural processing dysfunction. The FIE may be reflected at the electrophysiological level, with the N170 potential delayed and enhanced by an inverted face relative to an upright face in normal controls (McCarthy et al., 1999; Taylor et al., 2001; Itier and Taylor, 2002, 2004). To date, only one study has suggested that the FIE of N170 is significantly reduced in individuals with schizophrenia (Tsunoda et al., 2012). Although many studies have investigated the behavioral FIE deficit in people with schizophrenia, the FIE of the N170 in those with schizophrenia and its correlation with schizophrenia symptoms are unexplored.

The clinical symptoms of schizophrenia include positive symptoms, such as hallucinations and delusions, and negative symptoms, such as apathy and avolition. Whether there is a correlation between face-processing deficits and clinical symptoms in patients with schizophrenia remains controversial. Some behavior studies have shown that neither positive nor negative symptoms are accompanied by face discrimination performance (Addington and Addington, 1998; Baudouin et al., 2002; Caharel et al., 2007), whereas other studies find significant correlations between negative symptoms and face recognition performance (Sachs et al., 2004; Martin et al., 2005; Norton et al., 2009; Chen et al., 2012), and one study found significant correlations between both positive and negative symptoms and face identity performance (Chen et al., 2009). Although many studies have examined the correlation between face-processing behavioral indices and schizophrenic symptoms, few have explored the correlation between face-processing electrophysiological indices and schizophrenia symptoms. Thus, this correlation was assessed in the current study.

This study used a two (face, non-face) by two (upright, inverted) image design and electroencephalogram (EEG) recording to accomplish three goals. First, both the amplitude and latency of the N170 potential were measured in patients with schizophrenia and in healthy controls. Here, we tested the hypothesis that the face-specific N170 in patients with schizophrenia has smaller amplitude and enhanced delayed latency compared with those in healthy controls. Second, we measured the FIE of the N170 in these two populations to test the hypothesis that the FIE of $\mathrm{N} 170$ is absent in patients with schizophrenia. Finally, we assessed the correlation between the schizophrenia symptom scores and the N170 indices (including N170 amplitude, latency and FIE). Our overall aim was to determine whether N170 can be used an electrophysiological marker that objectively reflects the severity of schizophrenia.

\section{MATERIALS AND METHODS}

\section{Participants}

This study was approved by the Institutional Review Board of Guangzhou Brain Hospital. 24 patients with schizophrenia ( 12 women, mean age $32.3 \pm 11.2$ years old) and 24 age-matched healthy controls ( 12 women, mean age $32.9 \pm 11.5$ years old) participated in this study. All participants reported normal or corrected-to-normal vision. Each patient was diagnosed with schizophrenia according to the Diagnostic and Statistical Manual of Mental Disorders, Fourth Edition. Their psychiatric symptoms were evaluated by a trained psychiatrist or psychologist based on the Positive and Negative Syndrome Scale (Kay et al., 1987). The Personal and Social Performance scale, an acceptable, quick, and valid measure (Morosini et al., 2000), was conducted to assess participants' social functioning. No patient had a history of severe medical or severe neurological disorders. The demographic and descriptive characteristics of the participants are shown in Supplementary Table S1. No healthy volunteer reported a history of major psychiatric disorder or major physical illness or use of medication known to affect the central nervous system. The exclusion criteria for both groups included clear abnormality of MRI results, neurological illness, traumatic brain injury, substance abuse, or addiction. All participants received financial compensation and provided written informed consent for their participation and all the methods were performed in accordance with the clinical-experimental guidelines of human experiment approved by Institutional Review Board of Guangzhou Brain Hospital. No vulnerable populations were involved in this study.

\section{Stimuli and Procedure}

The stimuli were grayscale photographs $(10.58 \mathrm{~cm} \times 12.70 \mathrm{~cm})$ of 60 unfamiliar young faces, 60 tables and 62 flowers. Half of the faces were male, and all faces were presented without hair atop the head (facial hair was included), glasses, or other accessories (Figure 1). These stimuli were used to form five stimulus conditions: (a) upright faces; (b) upright tables; (c) inverted faces; (d) inverted tables; and (e) targets (flowers). All images were equated for luminance and root mean square contrast (calculations excluded the gray background) using Adobe Photoshop ${ }^{1}$ (Gao et al., 2009). The stimuli were presented

\footnotetext{
${ }^{1}$ www.adobe.com
} 


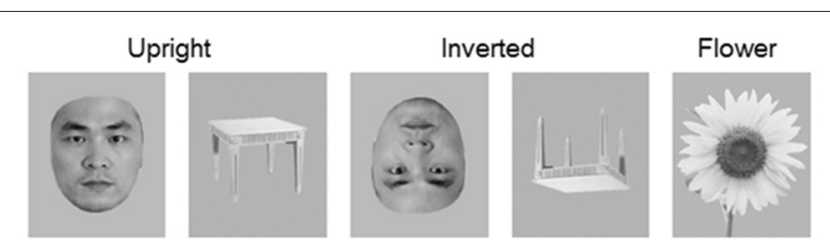

FIGURE 1 | Examples of target (flowers) and non-target stimuli (faces and tables) in upright and inverted orientations. Only the non-target stimulus-evoked event-related potential (ERP) were analyzed.

at fixation, and seen from a distance of $1.2 \mathrm{~m}$, they occupied a visual angle of $5.051^{\circ} \times 6.061^{\circ}$.

Participants were tested in a dimly lit room during the experiment. They sat in a comfortable chair and were instructed to maintain a mental count of the images of flowers they saw on the screen and to ignore all other stimuli. This procedure has been used in research examining the N170 component to assign similar task relevance to face and non-face stimuli (e.g., Carmel and Bentin, 2002; Gao et al., 2009). The 302 stimuli were presented one at a time in two blocks: one block comprised 20 upright faces, 20 inverted faces, 20 upright tables, 20 inverted tables and 20 flowers (targets); and the other block comprised 40 upright faces, 40 inverted faces, 40 upright tables, 40 inverted tables and 42 flowers (targets). The order of trials within each block was randomized. Stimulus exposure time was $250 \mathrm{~ms}$ and was separated by an inter-trial interval randomized from $750 \mathrm{~ms}$ to $1250 \mathrm{~ms}$. There was a 1-min break between blocks.

\section{Electrophysiological Recording}

The EEG was recorded continuously by a set of $16 \mathrm{Ag} / \mathrm{AgCl}$ electrodes placed according to the 10/20 system. The EEG recording sites were: F3, Fz, F4, C3, Cz, C4, P7, P3, Pz, P4, P8, $\mathrm{PO} 7, \mathrm{PO} 8, \mathrm{O} 1, \mathrm{Oz}$ and $\mathrm{O} 2$. An electrooculogram (EOG) was recorded to monitor eye movements and blinks via electrodes placed on bilateral external canthi and the left infraorbital and supraorbital areas. EEG and EOG signals were sampled at $1000 \mathrm{~Hz}$, with a $0.1-100 \mathrm{~Hz}$ bandpass filter using a NuAmps digital amplifier system (Neuroscan Labs, El Paso, TX, USA). The tip of the nose was used as a reference during recording, and determination of the approximate zero reference using the reference electrode standardization technique $\left(\mathrm{REST}^{2}\right)$ was conducted off-line (Yao, 2001; Tian and Yao, 2013). Electrode impedances were kept below $5 \mathrm{k} \Omega$.

\section{Data and Statistical Analyses}

The pre-processing of the electrophysiological data was conducted using the EEGLAB toolbox (Delorme and Makeig, 2004) in the MATLAB environment. The long-term EEG of each electrode was first bandpass filtered $(1-100 \mathrm{~Hz})$ and then segmented into epochs from $-100 \mathrm{~ms}$ to $400 \mathrm{~ms}$ around the onset. The baseline correction was conducted using a time window of $-100 \mathrm{~ms}$ to $0 \mathrm{~ms}$. Epochs that contained more than $\pm 1 \mathrm{mV}$ potential were rejected as artifacts. The remaining

${ }^{2}$ www.neuro.uestc.edu.cn/rest epochs were averaged to obtain an ERP for each electrode node and then low-pass filtered at $15 \mathrm{~Hz}$.

Statistical analysis was performed with IBM SPSS Statistics 20 software (SPSS Inc., Chicago, IL, USA). Analyses of variance (ANOVAs), $t$-tests and Spearman correlations were conducted. The $p$ values were corrected by Bonferroni adjustment for multiple comparisons. The null-hypothesis rejection level was set at 0.05 .

\section{RESULTS}

Target (flower) monitoring was equally great in both groups: $98.3 \%$ for patients with schizophrenia and 99.5\% for healthy controls ( $p>0.05$, independent samples $t$-test). Non-target stimuli (i.e., faces and tables) elicited clear P1 and N170 components, with significant temporo-occipital distribution in both the patient and healthy control groups (Supplementary Figure S1). Only the N170 component was further analyzed.

After comparing the REST and average reference (AR) results (Supplementary Tables S3, S5), we found that REST gave more significant results around the temporo-occipital area (Supplementary Figures S1, S2). Thus, the REST results are presented here, and the AR- and REST-based results are compared in the supplementary materials.

\section{N170 Latency and Amplitude}

As shown in Figure 2, the N170 latencies and amplitudes differed for the four experimental stimulus conditions in each group. Furthermore, the N170 waveform was generally lower in the patient group than in the control group. To evaluate the group and stimulus effects on the N170 potential, we conducted a threeway, mixed-measures ANOVAs for latencies and amplitudes: 2 (group effect: patient, control) $\times 2$ (Face effect: face, table $) \times 2$ (inversion effect: upright, inverted).

For the N170 latency analysis, significant group effects were found in O1, P7 and PO7 electrodes (all $p<0.05$ ) and significant inversion effects were found in P7, P8, and PO8 electrodes (all $p<0.05)$. A significant effect of face was found in P8 and PO8 (both $p<0.01$ ). A significant interaction for face $\times$ group was found in P8 $\left(F_{(1,46)}=4.674, p=0.036\right)$, and a significant interaction of face $\times$ inversion was found in PO7 $\left(F_{(1,46)}=6.918\right.$, $p=0.012$; details shown in Supplementary Table S2).

For the N170 amplitude analysis, a significant group effect was found only in $\mathrm{P} 7$ and $\mathrm{PO} 7$ electrodes (both $p<0.05$ ), and significant effect of face was found in $\mathrm{O} 1, \mathrm{O} 2, \mathrm{P} 7, \mathrm{P} 8, \mathrm{PO} 7$, $\mathrm{PO} 8$ and $\mathrm{Oz}$ electrodes (all $p<0.01$ ). No significant inversion effect was found. A significant interaction of face $\times$ inversion was found in P7 and PO7 (both $p<0.05$ ). No other significant interaction was observed in any electrode (details shown in Supplementary Table S4).

\section{N170 Group Differences}

As stated above, group effects for both N170 latency and amplitude were observed around the P7 electrode. To further analyze across group differences, we conducted independent 
A
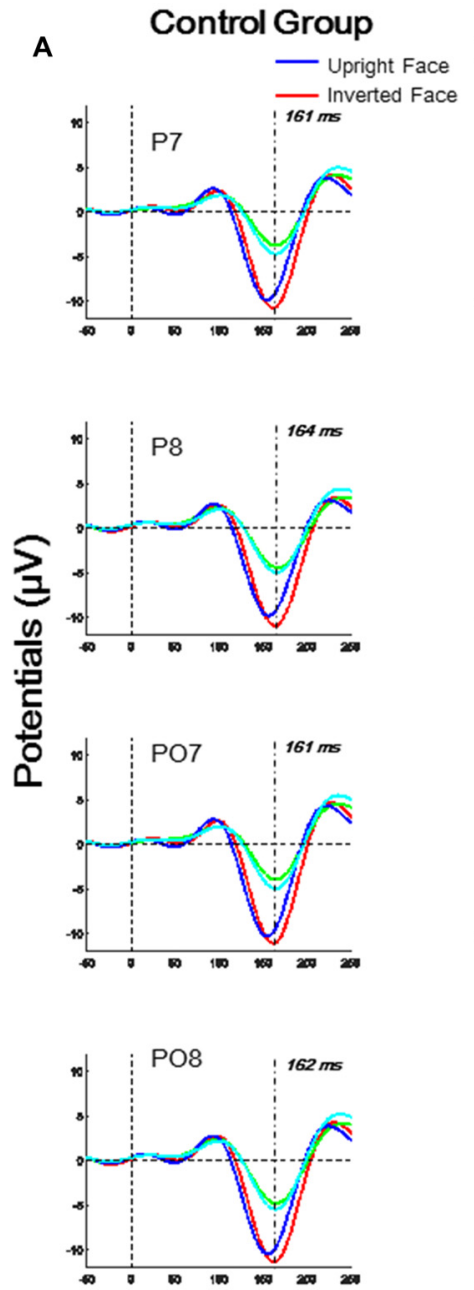

B

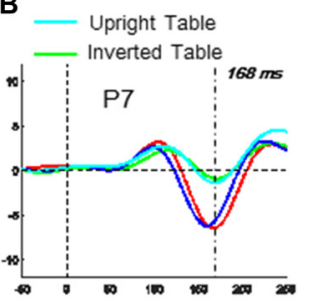

Patient Group
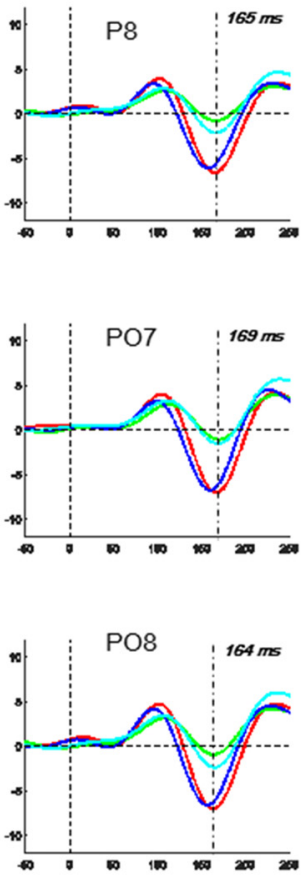

Time (ms)

FIGURE 2 | Temporo-occipital ERP waveforms (electrodes P7, P8, PO7, PO8) evoked using four stimulus conditions (upright and inverted images of faces and tables) in both control and patient groups.

(A) Control group; (B) Patient group.

samples $t$-tests between the patient and control groups for electrodes $\mathrm{P} 7, \mathrm{P} 8, \mathrm{PO} 7$ and $\mathrm{PO} 8$ for each of the four stimulus conditions. As shown in Figure 3A, the N170 peak latencies in patients were significantly longer than those in controls for both upright faces $\left(\mathrm{P} 7: t_{[46]}=3.036, p=0.004\right.$; PO7: $\left.t_{[46]}=2.861, p=0.006\right)$ and inverted faces $\left(\mathrm{P} 7: t_{[46]}=3.022\right.$, $p=0.004$; PO7: $\left.t_{[46]}=2.795, p=0.008\right)$ in the left hemisphere, without any significance difference in the right hemisphere (all $p>0.05$ for $\mathrm{P} 8$ and PO8). These results indicated sluggishness for the face-evoked N170 in patients with schizophrenia, with left hemisphere dominance.

As shown in Figure 3B, the N170 amplitude in patients was significantly smaller than that in controls for both inverted faces $\left(\mathrm{P} 7: t_{[46]}=2.432, p=0.019 ; \mathrm{PO} 7: t_{[46]}=2.223, p=0.031\right)$ and inverted tables (P7: $t_{[46]}=2.159, p=0.036$; PO7: $t_{[46]}=2.025$, $p=0.049)$ in the left hemisphere, while a significant difference

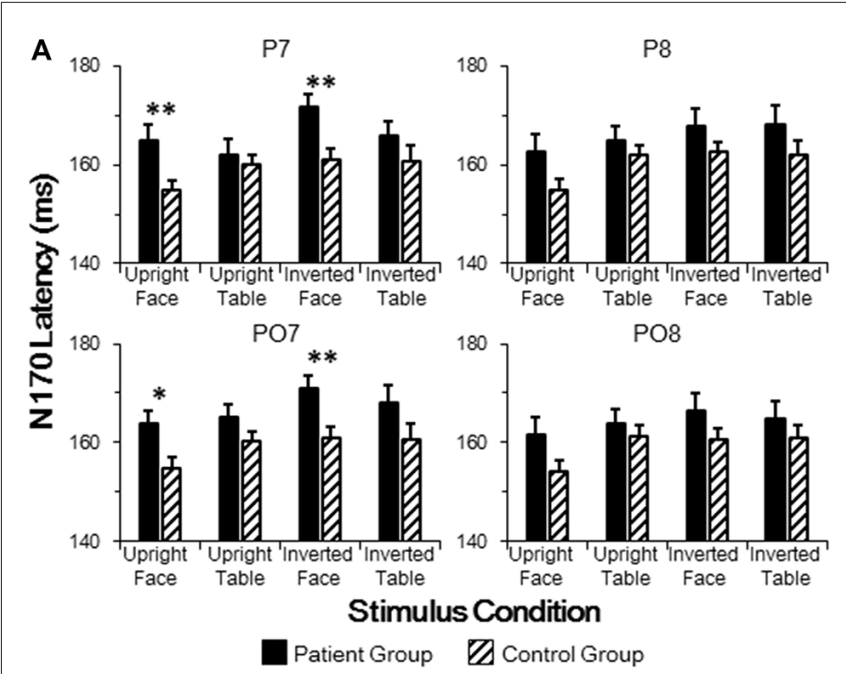

B

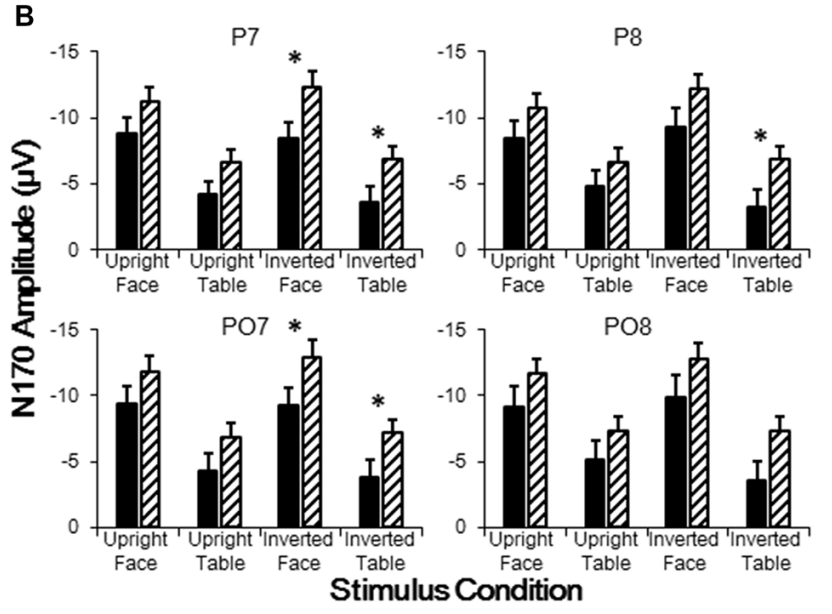

FIGURE 3 | Comparisons of N170 latencies (A) and amplitudes (B) in patient and control groups in temporo-occipital electrodes (P7, P8, P07 and PO8). Point lines: measurement of the peak latency when the face image was oriented upright. ${ }^{*} p<0.05,{ }^{* *} p<0.01$.

in the right hemisphere was found only for inverted tables at P8 $\left(t_{[46]}=2.100, p=0.041\right.$; all other comparisons $p>0.05$ for P8 and PO8). These results indicated that inverted face-evoked N170 amplitudes were reduced in patients with schizophrenia.

The differences between the stimulus conditions in each group were assessed using post hoc $t$-tests. In the control group, the N170 latency evoked by upright faces was significantly delayed compared with that evoked by upright tables (P8: $p=0.035$; PO7: $p=0.006$; PO8: $p=0.004$ ). However, in the patient group, the N170 latencies evoked by upright faces and upright tables were not significantly different (all $p>0.05$ ). In both control and patient groups, the N170 latency evoked by inverted faces was significantly larger than that evoked by upright faces (P7: both $p<0.001$; P8: both $p<0.001$; PO7: both $p<0.001$; PO8: both $p<0.001)$. No inversion effect was found for the table stimuli in either group (all $p>0.05$ ). In both control and patient groups, the N170 amplitudes evoked by faces were significantly larger than those evoked by either 
upright or inverted tables (P7: all $p<0.001$; P8: all $p<0.001$; PO7: all $p<0.001$; PO8: all $p<0.001$ ). In both groups, the N170 amplitude showed no significant difference between upright and inverted tables (all $p>0.05$ ).

\section{N170 Latency Correlated With Symptom Scores}

Spearman correlation tests were conducted for the N170 latencies and amplitudes (P7, P8, PO7 and PO8) of individual participants in both groups against the three clinical symptom scores, namely positive, negative and general psychiatric symptom scores.

The results showed that N170 latencies were correlated with negative symptom and general psychiatric symptom scores, with no significant correlation found between N170 latencies and positive symptom scores. To be more specific, the upright face-evoked N170 latencies in P7 and PO7 were significantly and positively correlated with general psychiatric symptom scores (both $p<0.05$; Figure 4B), and the inverted face-evoked N170 latencies in P7 and PO7 were significantly and positively correlated with both negative symptom scores (Figure 4A) and general psychiatric symptom scores (all $p<0.05$; Figure 4B). Thus, significant correlations were found only for face stimuli, not for table stimuli. No significant correlation was found in P8 or PO8 between any symptom and stimulus condition. Similarly, no significant correlation was found between the N170 amplitude and any symptom score. These results indicated that N170 latency sluggishness may effectively predict clinical symptom scores at the level of the individual.

\section{Abnormal Face Inversion Effect}

To evaluate whether upright and inverted faces were differentially processed in the patient and control groups, we calculated FIE scores for each participant, which was defined as N170 amplitudes evoked by upright faces minus those evoked by inverted faces. One-sample $t$-tests were used to determine whether the FIE score was significantly larger than 0 . We found that the FIE scores in the control group were significantly larger than 0 (P7: $t_{[23]}=2.484, p=0.021 ; \mathrm{P} 8: t_{[23]}=3.679$, $p=0.001$; PO7: $t_{[23]}=2.232, p=0.036$; PO8: $t_{[23]}=2.753$, $p=0.011$ ), whereas the FIE scores in the patient group were not significantly different from 0 (Supplementary Figure S3). However, no significant correlation was found using Spearman correlation tests between FIE and symptom scores. These results demonstrated abnormality of holistic face processing in patients with schizophrenia.

\section{DISCUSSION}

\section{Face-Specific Deficits in Schizophrenia}

The dysfunction of non-emotional face processing in patients with schizophrenia is controversial because such a deficit in these patients is not always specific to faces (for reviews see Darke et al., 2013; Onitsuka et al., 2013; Watson, 2013; Bortolon et al., 2015; McCleery et al., 2015). Although the face-specific area is mainly localized to the fusiform gyrus, neurophysiological studies have reported decreased activities (Desco et al., 2003;

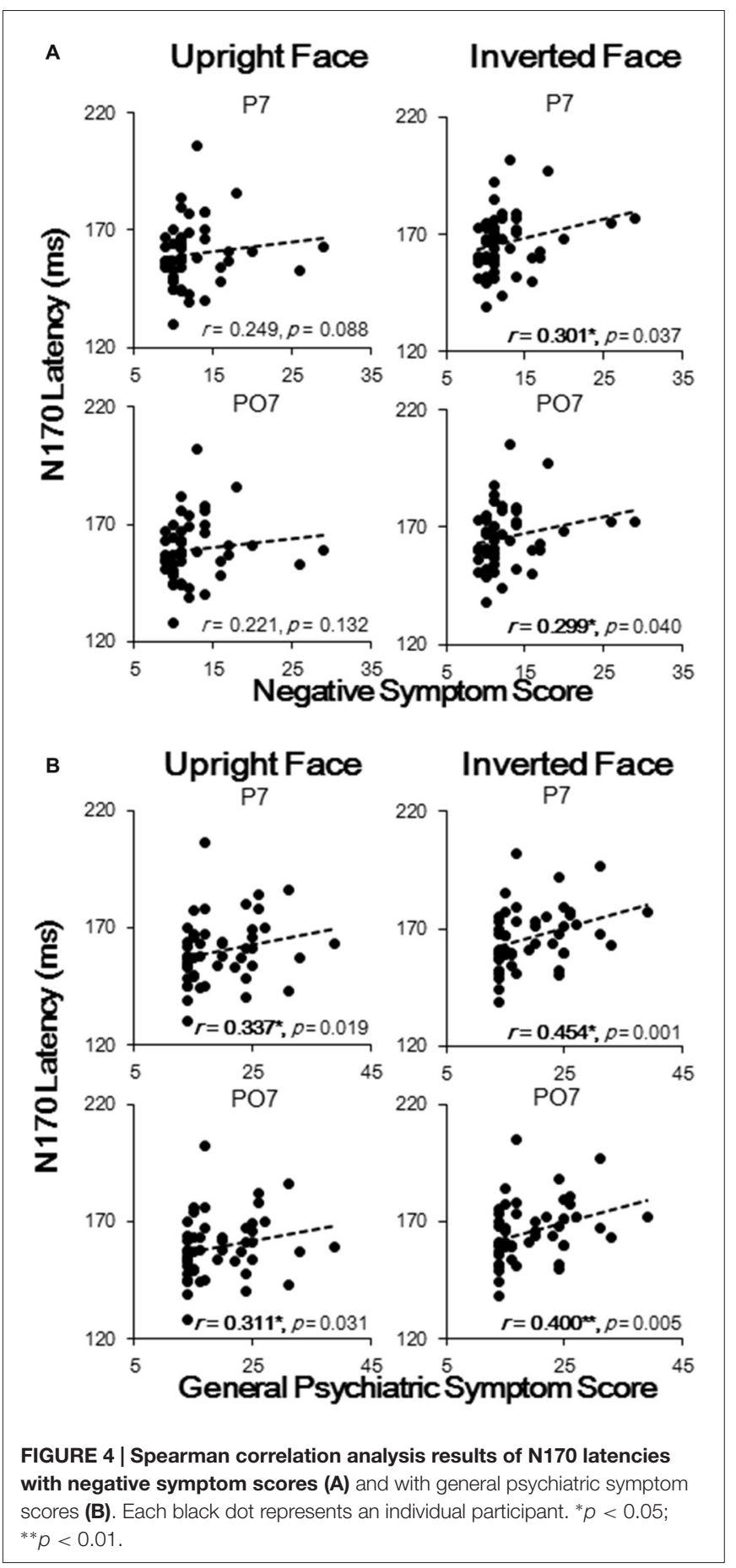

Silverstein et al., 2010) and reductions in the total neuron number (Dorph-Petersen et al., 2007) in the early visual cortex (V1-V3) in patients with schizophrenia. To extract the face-specific components for holistic processing, we used images of both upright and inverted faces together with non-face images to generate N170 ERPs in both patients with schizophrenia and healthy controls.

As shown in Figure 3, the N170 component was reduced and delayed in patients compared with that in healthy controls, 
which is consistent with the results of previous ERP studies (Herrmann et al., 2004; Onitsuka et al., 2006; Tsunoda et al., 2012). More specifically, in patients with schizophrenia, the N170 latencies were delayed only under face-evoked conditions (both upright and inverted). By contrast, the decrease in the N170 amplitudes showed no face selectivity. In addition, the face-evoked N170 latencies were positively correlated with negative symptom scores and general psychiatric symptom scores (Figure 4). No significant correlations were found for N170 latencies under non-face-evoked conditions, indicating that the face-specific N170 latencies could, to a certain extent, predict the severity of schizophrenia symptoms. Therefore, the face-specific N170 latency may be a useful neurophysiological index of some symptoms in patients with schizophrenia.

Neurophysiological function in schizophrenia is thought to be asymmetrical across hemispheres (Crow et al., 1989; Gruzelier, 1999). In this study, the face-specific N170 latency was delayed only in the left hemisphere of patients with schizophrenia. This hemisphere asymmetry is further supported by structural studies that have identified a smaller left fusiform gyrus gray matter volume in schizophrenic patients compared with those in healthy controls (McDonald et al., 2000; Lee et al., 2002). These results demonstrated a left hemisphere involvement of face-specific dysfunction in patients with schizophrenia.

\section{Face Inversion Effect Absence in Schizophrenia}

A major hypothesis for the processing of global face configurations requires that additional areas be involved in the processed (Eimer, 2000a,b; Itier and Taylor, 2004), which is supported by an ERP dipole analysis study (Watanabe et al., 2003) and an ERP competition paradigm (Sadeh and Yovel, 2010). Moreover, an fMRI study revealed that inverted face processing recruits regions involved in face or object processing (Haxby et al., 1999). Rosburg et al. (2010) illustrated that the lateral occipital cortex contributes to the FIE of ERPs. In our study (shown in Supplementary Figure S2), significant increases in the N170 amplitude evoked by inverted faces compared with those by upright faces (i.e., the FIE) were observed only in healthy controls, not in patients with schizophrenia, which is consistent with the results of a previous study (Tsunoda et al., 2012). However, we found no significant correlation between the FIE and any clinical symptom. These results suggest that the face-selective cortex may be associated with the pathophysiology in schizophrenia. According to the study by Tsunoda et al. (2012), the FIE is absent in patients with schizophrenia, which might be related to social function. Thus, the abnormality of neural responses in areas related to holistic face processing may have a close relationship to social dysfunction but not to clinical symptoms.

\section{The Demyelination Hypothesis of Schizophrenia}

In this study, face-specific N170 latency was delayed in the patient group, and this sluggishness was positively correlated with schizophrenia symptoms (Figures 3, 4). These findings lead to the hypothesis that a common neural mechanism underlies both clinical symptoms and face-processing defects in patients with schizophrenia.

One of the most popular perspectives accounting for the neuropathology observed in schizophrenia is the demyelination hypothesis (Chance et al., 1999; Foong et al., 2001; Hakak et al., 2001; Uranova et al., 2001; Davis et al., 2003; Flynn et al., 2003; Kubicki et al., 2005), which can explain the white matter damage (Okugawa et al., 2002; Federspiel et al., 2006; DeLisi, 2008; Friedman et al., 2008; Gasparotti et al., 2009), brain network synchrony reductions (Spencer et al., 2003, 2004; McIntosh et al., 2008; Uhlhaas et al., 2008; Peters et al., 2010; Uhlhaas and Singer, 2010), and genetic defects (Hakak et al., 2001; McCullumsmith et al., 2007) in schizophrenic patients with a simple myelin-related dysfunction mechanism.

Previous studies have shown that myelin-related defects are relevant to schizophrenia symptoms (Sanfilipo et al., 2000; Sigmundsson et al., 2001), and damage to myelin can result in conduction delays in neural discharge (Whitford et al., 2012). For instance, the visual-evoked P1 components in patients with multiple sclerosis are delayed about $20 \mathrm{~ms}$ because of damage to the optic nerve myelin (Cowan et al., 1984; Brusa et al., 2001). In the current study, the face-specific N170 latency was delayed in the patient group compared with that in healthy controls, and this sluggishness was positively correlated with negative symptoms and general psychiatric symptoms, providing additional evidence supporting the demyelination hypothesis of schizophrenia.

\section{Limitations}

Although this study revealed a face-specific processing delay in patients with schizophrenia that was correlated with clinical symptoms, the key brain structure corresponding to this delay was not determined. Simultaneous measurement of the ERP and $\mathrm{FMRI}$ in future studies may provide more precise temporal dynamics and spatial locus of impaired face-specific processing in schizophrenia. This study only discussed the results relative to the demyelination hypothesis of schizophrenia; advanced MRI technology could be used in future studies to provide a myelin density map for further validation (Glasser et al., 2016).

\section{CONCLUSION}

This study investigated face-specific neurophysiological markers and their relationships with clinical symptoms in people with schizophrenia and in healthy controls. Compared with healthy controls, significant N170 latency sluggishness was found in the schizophrenia group. This neural timing sluggishness increased with the severity of both negative and general mental symptoms, suggesting a correlation between dysfunctions in face-specific processing and the development of symptoms in people with schizophrenia. 


\section{AUTHOR CONTRIBUTIONS}

YZ, HL, YN, JR, ZW, RH, TL, GL, TB, QW and SS contributed to the design of the study and wrote protocols. The experiments were conducted by YZ, HL and SS. QW wrote the first draft of the manuscript. All authors contributed to and approved the final manuscript.

\section{ACKNOWLEDGMENTS}

$\mathrm{YZ}$ and HL contributed equally to this manuscript. We thank Zhemeng $\mathrm{Wu}$ for her comments on the manuscript. This work was supported by National Natural Science Foundation of China $(81671334,31400960,81571275)$, the Planned Science

\section{REFERENCES}

Addington, J., and Addington, D. (1998). Facial affect recognition and information processing in schizophrenia and bipolar disorder. Schizophr. Res. 32, 171-181. doi: 10.1016/s0920-9964(98)00042-5

Baudouin, J.-Y., Martin, F., Tiberghien, G., Verlut, I., and Franck, N. (2002). Selective attention to facial emotion and identity in schizophrenia. Neuropsychologia 40, 503-511. doi: 10.1016/s0028-3932(01)00114-2

Bauser, D. S., Thoma, P., Aizenberg, V., Brüne, M., Juckel, G., and Daum, I. (2012). Face and body perception in schizophrenia: a configural processing deficit? Psychiatry Res. 195, 9-17. doi: 10.1016/j.psychres.2011.07.017

Bentin, S., Allison, T., Puce, A., Perez, E., and McCarthy, G. (1996). Electrophysiological studies of face perception in humans. J. Cogn. Neurosci. 8, 551-565. doi: 10.1162/jocn.1996.8.6.551

Bortolon, C., Capdevielle, D., and Raffard, S. (2015). Face recognition in schizophrenia disorder: a comprehensive review of behavioral, neuroimaging and neurophysiological studies. Neurosci. Biobehav. Rev. 53, 79-107. doi: 10. 1016/j.neubiorev.2015.03.006

Brusa, A., Jones, S. J., and Plant, G. T. (2001). Long-term remyelination after optic neuritis: a 2-year visual evoked potential and psychophysical serial study. Brain 124, 468-479. doi: 10.1093/brain/124.3.468

Caharel, S., Bernard, C., Thibaut, F., Haouzir, S., Di Maggio-Clozel, C., Allio, G., et al. (2007). The effects of familiarity and emotional expression on face processing examined by ERPs in patients with schizophrenia. Schizophr. Res. 95, 186-196. doi: 10.1016/j.schres.2007.06.015

Carmel, D., and Bentin, S. (2002). Domain specificity versus expertise: factors influencing distinct processing of faces. Cognition 83, 1-29. doi: 10.1016/s00100277(01)00162-7

Chance, S. A., Highley, J. R., Esiri, M. M., and Crow, T. J. (1999). Fiber content of the fornix in schizophrenia: lack of evidence for a primary limbic encephalopathy. Am. J. Psychiatry 156, 1720-1724.

Chen, Y. (2011). Abnormal visual motion processing in schizophrenia: a review of research progress. Schizophr. Bull. 37, 709-715. doi: 10.1093/schbul/sbr020

Chen, Y., Cataldo, A., Norton, D. J., and Ongur, D. (2012). Distinct facial processing in schizophrenia and schizoaffective disorders. Schizophr. Res. 134, 95-100. doi: 10.1016/j.schres.2011.08.001

Chen, Y., Norton, D., McBain, R., Ongur, D., and Heckers, S. (2009). Visual and cognitive processing of face information in schizophrenia: detection, discrimination and working memory. Schizophr. Res. 107, 92-98. doi: 10.1016/j. schres.2008.09.010

Cowan, J. M. A., Dick, J. P. R., Day, B. L., Rothwell, J. C., Thompson, P. D., and Marsden, C. D. (1984). Abnormalities in central motor pathway conduction in multiple sclerosis. Lancet 324, 304-307. doi: 10.1016/s0140-6736(84) 92683-7

Crow, T. J., Ball, J., Bloom, S. R., Brown, R., Bruton, C. J., Colter, N., et al. (1989). Schizophrenia as an anomaly of development of cerebral asymmetry: a postmortem study and a proposal concerning the genetic basis of the disease. Arch. Gen. Psychiatry 46, 1145-1150. doi: 10.1001/archpsyc.1989. 01810120087013 and Technology Projects of Guangzhou (2014Y2-00105), the Guangzhou Municipal Key Discipline in Medicine for Guangzhou Brain Hospital (GBH2014-ZD06, GBH2014-QN04), Scientific Research Common Program of Beijing Commission of Education (KM201410025027), the Capital Health Research and Development of Special Found (2016-1-8012) and Beijing Municipal Science and Technology Commission (Z161100000516230).

\section{SUPPLEMENTARY MATERIAL}

The Supplementary Material for this article can be found online at: http://journal.frontiersin.org/article/10.3389/fnhum. 2016.00615/full\#supplementary-material

Darke, H., Peterman, J. S., Park, S., Sundram, S., and Carter, O. (2013). Are patients with schizophrenia impaired in processing non-emotional features of human faces? Front. Psychol. 4:529. doi: 10.3389/fpsyg.2013.00529

Davis, K. L., Stewart, D. G., Friedman, J. I., Buchsbaum, M., Harvey, P. D., Hof, P. R., et al. (2003). White matter changes in schizophrenia: evidence for myelin-related dysfunction. Arch. Gen. Psychiatry 60, 443-456. doi: 10. 1001/archpsyc.60.5.443

DeLisi, L. E. (2008). The concept of progressive brain change in schizophrenia: implications for understanding schizophrenia. Schizophr. Bull. 34, 312-321. doi: $10.1093 / \mathrm{schbul} / \mathrm{sbm} 164$

Delorme, A., and Makeig, S. (2004). EEGLAB: an open source toolbox for analysis of single-trial EEG dynamics including independent component analysis. J. Neurosci. Methods 134, 9-21. doi: 10.1016/j.jneumeth.2003.10.009

Desco, M., Gispert, J. D., Reig, S., Sanz, J., Pascau, J., Sarramea, F., et al. (2003). Cerebral metabolic patterns in chronic and recent-onset schizophrenia. Psychiatry Res. 122, 125-135. doi: 10.1016/s0925-4927(02)00124-5

Dorph-Petersen, K. A., Pierri, J. N., Wu, Q., Sampson, A. R., and Lewis, D. A. (2007). Primary visual cortex volume and total neuron number are reduced in schizophrenia. J. Comp. Neurol. 501, 290-301. doi: 10.1002/cne. 21243

Eimer, M. (2000a). Event-related brain potentials distinguish processing stages involved in face perception and recognition. Clin. Neurophysiol. 111, 694-705. doi: 10.1016/s1388-2457(99)00285-0

Eimer, M. (2000b). The face-specific N170 component reflects late stages in the structural encoding of faces. Neuroreport 11, 2319-2324. doi: 10. 1097/00001756-200007140-00050

Federspiel, A., Begré, S., Kiefer, C., Schroth, G., Strik, W. K., and Dierks, T. (2006). Alterations of white matter connectivity in first episode schizophrenia. Neurobiol. Dis. 22, 702-709. doi: 10.1016/j.nbd.2006.01.015

Flynn, S. W., Lang, D. J., Mackay, A. L., Goghari, V., Vavasour, I. M., Whittall, K. P., et al. (2003). Abnormalities of myelination in schizophrenia detected in vivo with MRI and post-mortem with analysis of oligodendrocyte proteins. Mol. Psychiatry 8, 811-820. doi: 10.1038/sj.mp. 4001337

Foong, J., Symms, M. R., Barker, G. J., Maier, M., Woermann, F. G., Miller, D. H. et al. (2001). Neuropathological abnormalities in schizophrenia: evidence from magnetization transfer imaging. Brain 124, 882-892. doi: 10.1093/brain/124. 5.882

Friedman, J. I., Tang, C., Carpenter, D., Buchsbaum, M., Schmeidler, J., Flanagan, L., et al. (2008). Diffusion tensor imaging findings in first-episode and chronic schizophrenic patients. Am. J. Psychiatry 165, 1024-1032. doi: 10. 1176/appi.ajp.2008.07101640

Gao, L., Xu, J., Zhang, B., Zhao, L., Harel, A., and Bentin, S. (2009). Aging effects on early-stage face perception: an ERP study. Psychophysiology 46, 970-983. doi: 10.1111/j.1469-8986.2009.00853.x

Gasparotti, R., Valsecchi, P., Carletti, F., Galluzzo, A., Liserre, R., Cesana, B. et al. (2009). Reduced fractional anisotropy of corpus callosum in first-contact, antipsychotic drug-naive patients with schizophrenia. Schizophr. Res. 108, 41-48. doi: 10.1016/j.schres.2008.11.015 
Glasser, M. F., Coalson, T., Robinson, E., Hacker, C., Harwell, J., Yacoub, E., et al. (2016). A multi-modal parcellation of human cerebral cortex. Nature 536, 171-178. doi: 10.1038/nature 18933

Gruzelier, J. H. (1999). Functional neuropsychophysiological asymmetry in schizophrenia: a review and reorientation. Schizophr. Bull. 25, 91-120. doi: 10. 1093/oxfordjournals.schbul.a033370

Hakak, Y., Walker, J. R., Li, C., Wong, W. H., Davis, K. L., Buxbaum, J. D., et al. (2001). Genome-wide expression analysis reveals dysregulation of myelinationrelated genes in chronic schizophrenia. Proc. Natl. Acad. Sci. U S A 98, 4746-4751. doi: 10.1073/pnas.081071198

Haxby, J. V., Ungerleider, L. G., Clark, V. P., Schouten, J. L., Hoffman, E. A., and Martin, A. (1999). The effect of face inversion on activity in human neural systems for face and object perception. Neuron 22, 189-199. doi: 10 . 1016/s0896-6273(00)80690-x

Herrmann, M. J., Ellgring, H., and Fallgatter, A. J. (2004). Early-stage face processing dysfunction in patients with schizophrenia. Am. J. Psychiatry 161, 915-917. doi: 10.1176/appi.ajp.161.5.915

Itier, R. J., and Taylor, M. J. (2002). Inversion and contrast polarity reversal affect both encoding and recognition processes of unfamiliar faces: a repetition study using ERPs. Neuroimage 15, 353-372. doi: 10.1006/nimg.2001.0982

Itier, R. J., and Taylor, M. J. (2004). N170 or N1? Spatiotemporal differences between object and face processing using ERPs. Cereb. Cortex 14, 132-142. doi: 10.1093/cercor/bhg111

Kay, S. R., Flszbein, A., and Opfer, L. A. (1987). The positive and negative syndrome scale (PANSS) for schizophrenia. Schizophr. Bull. 13, 261-276. doi: 10.1093/schbul/13.2.261

Kim, H. S., Shin, N. Y., Choi, J. S., Jung, M. H., Jang, J. H., Kang, D. H., et al. (2010). Processing of facial configuration in individuals at ultra-high risk for schizophrenia. Schizophr. Res. 118, 81-87. doi: 10.1016/j.schres.2010.01.003

Kubicki, M., Park, H., Westin, C. F., Nestor, P. G., Mulkern, R. V., Maier, S. E., et al. (2005). DTI and MTR abnormalities in schizophrenia: analysis of white matter integrity. Neuroimage 26, 1109-1118. doi: 10.1016/j.neuroimage.2005. 03.026

Lee, C. U., Shenton, M. E., Salisbury, D. F., Kasai, K., Onitsuka, T., Dickey, C. C., et al. (2002). Fusiform gyrus volume reduction in first-episode schizophrenia: a magnetic resonance imaging study. Arch. Gen. Psychiatry 59, 775-781. doi: 10. 1001/archpsyc.59.9.775

Maher, S., Ekstrom, T., Holt, D., Ongur, D., and Chen, Y. (2015). The core brain region for face processing in schizophrenia lacks face selectivity. Schizophr. Bull. 42, 666-674. doi: 10.1093/schbul/sbv140

Maher, S., Mashhoon, Y., Ekstrom, T., Lukas, S., and Chen, Y. (2016). Deficient cortical face-sensitive N170 responses and basic visual processing in schizophrenia. Schizophr. Res. 170, 87-94. doi: 10.1016/j.schres.2015.12.005

Martin, F., Baudouin, J. Y., Tiberghien, G., and Franck, N. (2005). Processing emotional expression and facial identity in schizophrenia. Psychiatry Res. 134, 43-53. doi: 10.1016/j.psychres.2003.12.031

McCleery, A., Lee, J., Joshi, A., Wynn, J. K., Hellemann, G. S., and Green, M. F. (2015). Meta-analysis of face processing event-related potentials in schizophrenia. Biol. Psychiatry 77, 116-126. doi: 10.1016/j.biopsych.2014.04. 015

McCarthy, G., Puce, A., Belger, A., and Allison, T. (1999). Electrophysiological studies of human face perception. II: response properties of face-specific potentials generated in occipitotemporal cortex. Cereb. Cortex 9, 431-444. doi: $10.1093 /$ cercor/9.5.431

McCullumsmith, R. E., Gupta, D., Beneyto, M., Kreger, E., Haroutunian, V., Davis, K. L., et al. (2007). Expression of transcripts for myelination-related genes in the anterior cingulate cortex in schizophrenia. Schizophr. Res. 90, 15-27. doi: 10.1016/j.schres.2006.11.017

McDonald, B., Highley, J. R., Walker, M. A., Herron, B. M., Cooper, S. J., Esiri, M. M., et al. (2000). Anomalous asymmetry of fusiform and parahippocampal gyrus gray matter in schizophrenia: a postmortem study. Am. J. Psychiatry 157, 40-47. doi: 10.1176/ajp.157.1.40

McIntosh, A. M., Muñoz Maniega, S., Lymer, G. K., McKirdy, J., Hall, J., Sussmann, J. E., et al. (2008). White matter tractography in bipolar disorder and schizophrenia. Biol. Psychiatry 64, 1088-1092. doi: 10.1016/j.biopsych.2008. 07.026

Megreya, A. M. (2016). Face perception in schizophrenia: a specific deficit. Cogn. Neuropsychiatry 21, 60-72. doi: 10.1080/13546805.2015.1133407
Morosini, P. L., Magliano, L., Brambilla, L., Ugolini, S., and Pioli, R. (2000). Development, reliability and acceptability of a new version of the DSM-IV Social and Occupational Functioning Assessment Scale (SOFAS) to assess routine social funtioning. Acta Psychiat. Scand. 101, 323-329. doi: 10.1111/j. 1600-0447.2000.tb10933.x

Norton, D., McBain, R., Holt, D. J., Ongur, D., and Chen, Y. (2009). Association of impaired facial affect recognition with basic facial and visual processing deficits in schizophrenia. Biol. Psychiatry 65, 1094-1098. doi: 10.1016/j.biopsych.2009. 01.026

Okugawa, G., Sedvall, G. C., and Agartz, I. (2002). Reduced grey and white matter volumes in the temporal lobe of male patients with chronic schizophrenia. Eur. Arch. Psychiatry Clin. Neurosci. 252, 120-123. doi: 10.1007/s00406-0020370-9

Onitsuka, T., Niznikiewicz, M. A., Spencer, K. M., Frumin, M., Kuroki, N., Lucia, L. C., et al. (2006). Functional and structural deficits in brain regions subserving face perception in schizophrenia. Am. J. Psychiatry 163, 455-462. doi: 10.1176/appi.ajp.163.3.455

Onitsuka, T., Oribe, N., Nakamura, I., and Kanba, S. (2013). Review of neurophysiological findings in patients with schizophrenia. Psychiatry Clin Neurosci. 67, 461-470. doi: 10.1111/pcn.12090

Peters, B. D., Blaas, J., and de Haan, L. (2010). Diffusion tensor imaging in the early phase of schizophrenia: what have we learned? J. Psychiat. Res 44, 993-1004. doi: 10.1016/j.jpsychires.2010.05.003

Rosburg, T., Ludowig, E., Dümpelmann, M., Alba-Ferrara, L., Urbach, H., and Elger, C. E. (2010). The effect of face inversion on intracranial and scalp recordings of event-related potentials. Psychophysiology 47, 147-157. doi: 10. 1111/j.1469-8986.2009.00881.x

Rossion, B., Caldara, R., Seghier, M., Schuller, A. M., Lazeyras, F., and Mayer, E. (2003). A network of occipito-temporal face-sensitive areas besides the right middle fusiform gyrus is necessary for normal face processing. Brain 126, 2381-2395. doi: 10.1093/brain/awg241

Sachs, G., Steger-Wuchse, D., Kryspin-Exner, I., Gur, R. C., and Katschnig, H. (2004). Facial recognition deficits and cognition in schizophrenia. Schizophr. Res. 68, 27-35. doi: 10.1016/s0920-9964(03)00131-2

Sadeh, B., and Yovel, G. (2010). Why is the N170 enhanced for inverted faces? An ERP competition experiment. Neuroimage 53, 782-789. doi: 10.1016/j. neuroimage.2010.06.029

Sanfilipo, M., Lafargue, T., Rusinek, H., Arena, L., Loneragan, C., Lautin, A., et al. (2000). Volumetric measure of the frontal and temporal lobe regions in schizophrenia: relationship to negative symptoms. Arch. Gen. Psychiatry 57, 471-480. doi: 10.1001/archpsyc.57.5.471

Shin, Y. W., Na, M. H., Ha, T. H., Kang, D. H., Yoo, S. Y., and Kwon, J. S. (2008). Dysfunction in configural face processing in patients with schizophrenia. Schizophr. Bull. 34, 538-543. doi: 10.1093/schbul/sbm118

Sigmundsson, T., Suckling, J., Maier, M., Williams, S., Bullmore, E. Greenwood, K., et al. (2001). Structural abnormalities in frontal, temporal and limbic regions and interconnecting white matter tracts in schizophrenic patients with prominent negative symptoms. Am. J. Psychiatry 158, 234-243. doi: 10.1176/appi.ajp.158.2.234

Silverstein, S. M., All, S. D., Kasi, R., Berten, S., Essex, B., Lathrop, K. L., et al. (2010). Increased fusiform area activation in schizophrenia during processing of spatial frequency-degraded faces, as revealed by fMRI. Psychol. Med. 40, 1159-1169. doi: 10.1017/S0033291709991735

Spencer, K. M., Nestor, P. G., Niznikiewicz, M. A., Salisbury, D. F., Shenton, M. E. and McCarley, R. W. (2003). Abnormal neural synchrony in schizophrenia. J. Neurosci. 23, 7407-7411.

Spencer, K. M., Nestor, P. G., Perlmutter, R., Niznikiewicz, M. A., Klump, M. C., Frumin, M., et al. (2004). Neural synchrony indexes disordered perception and cognition in schizophrenia. Proc. Natl. Acad. Sci. U S A 101, 17288-17293. doi: 10.1073/pnas.0406074101

Taylor, M. J., Edmonds, G. E., McCarthy, G., and Allison, T. (2001). Eyes first! Eye processing develops before face processing in children. Neuroreport 12, 1671-1676. doi: 10.1097/00001756-200106130-00031

Tian, Y., and Yao, D. (2013). Why do we need to use a zero reference? Reference influences on the ERPs of audiovisual effects. Psychophysiology 50, 1282-1290. doi: $10.1111 /$ psyp. 12130

Tsunoda, T., Kanba, S., Ueno, T., Hirano, Y., Hirano, S., Maekawa, T., et al. (2012). Altered face inversion effect and association between face N170 reduction 
and social dysfunction in patients with schizophrenia. Clin. Neurophysiol. 123, 1762-1768. doi: 10.1016/j.clinph.2012.01.024

Uhlhaas, P. J., Haenschel, C., Nikolić, D., and Singer, W. (2008). The role of oscillations and synchrony in cortical networks and their putative relevance for the pathophysiology of schizophrenia. Schizophr. Bull. 34, 927-943. doi: 10. 1093/schbul/sbn062

Uhlhaas, P. J., and Singer, W. (2010). Abnormal neural oscillations and synchrony in schizophrenia. Nat. Rev. Neurosci. 11, 100-113. doi: 10.1038/nrn2774

Uranova, N., Orlovskaya, D., Vikhreva, O., Zimina, I., Kolomeets, N., Vostrikov, V., et al. (2001). Electron microscopy of oligodendroglia in severe mental illness. Brain. Res. Bull. 55, 597-610. doi: 10.1016/s03619230(01)00528-7

Watanabe, S., Kakigi, R., and Puce, A. (2003). The spatiotemporal dynamics of the face inversion effect: a magneto- and electro-encephalographic study. Neuroscience 116, 879-895. doi: 10.1016/s0306-4522(02)00752-2

Watson, T. L. (2013). Implications of holistic face processing in autism and schizophrenia. Front. Psychol. 4:414. doi: 10.3389/fpsyg.2013.00414

Whitford, T. J., Ford, J. M., Mathalon, D. H., Kubicki, M., and Shenton, M. E. (2012). Schizophrenia, myelination and delayed corollary discharges: a hypothesis. Schizophr. Bull. 38, 486-494. doi: 10.1093/schbul/sbq105
Yao, D. (2001). A method to standardize a reference of scalp EEG recordings to a point at infinity. Physiol. Meas. 22, 693-711. doi: 10.1088/0967-3334/22/ $4 / 305$

Yin, R. K. (1969). Looking at upside-down faces. J. Exp. Psychol. 81, 141-145. doi: $10.1037 / \mathrm{h} 0027474$

Yin, R. K. (1970). Face recognition by brain-injured patients: a dissociable ability? Neuropsychologia 8, 395-402. doi: 10.1016/0028-3932(70) 90036-9

Conflict of Interest Statement: The authors declare that the research was conducted in the absence of any commercial or financial relationships that could be construed as a potential conflict of interest.

Copyright $\odot 2016$ Zheng, Li, Ning, Ren, Wu, Huang, Luan, Li, Bi, Wang and She. This is an open-access article distributed under the terms of the Creative Commons Attribution License (CC BY). The use, distribution and reproduction in other forums is permitted, provided the original author(s) or licensor are credited and that the original publication in this journal is cited, in accordance with accepted academic practice. No use, distribution or reproduction is permitted which does not comply with these terms. 